\title{
Limited Service Availability, Readiness, and Use of Facility- Based Delivery Care in Haiti: A Study Linking Health Facility Data and Population Data
}

\author{
Wenjuan Wang, ${ }^{a}$ Michelle Winner, ${ }^{a}$ Clara R Burgert-Brucker ${ }^{a}$
}

Proximity to a health facility offering delivery services and readiness of the facilities to provide such services were poor in both rural and urban areas outside of Port-au-Prince. Availability of a proximate facility was significantly associated with women in rural and urban areas delivering at a facility, as was the quality of delivery care available at the facilities but only in urban areas.

\section{ABSTRACT}

Background: Understanding the barriers that women in Haiti face to giving birth at a health facility is important for improving coverage of facility delivery and reducing persistently high maternal mortality. We linked health facility survey data and population survey data to assess the role of the obstetric service environment in affecting women's use of facility delivery care.

Methods: Data came from the 2012 Haiti Demographic and Health Survey (DHS) and the 2013 Haiti Service Provision Assessment (SPA) survey. DHS clusters and SPA facilities were linked with their geographic coordinate information. The final analysis sample from the DHS comprised 4,921 women who had a live birth in the 5 years preceding the survey. Service availability was measured with the number of facilities providing delivery services within a specified distance from the cluster (within 5 kilometers for urban areas and 10 kilometers for rural areas). We measured facility readiness to provide obstetric care using 37 indicators defined by the World Health Organization. Random-intercept logistic regressions were used to model the variation in individual use of facility-based delivery care and cluster-level service availability and readiness, adjusting for other factors.

Results: Overall, $39 \%$ of women delivered their most recent birth at a health facility and $61 \%$ delivered at home, with disparities by residence (about $60 \%$ delivered at a health facility in urban areas vs. $24 \%$ in rural areas). About one-fifth $(18 \%)$ of women in rural areas and one-tenth $(12 \%)$ of women in nonmetropolitan urban areas lived in clusters where no facility offered delivery care within the specified distances, while nearly all women (99\%) in the metropolitan area lived in clusters that had at least 2 such facilities. Urban clusters had better service readiness compared with rural clusters, with a wide range of variation in both areas. Regression models indicated that in both rural and nonmetropolitan urban areas availability of delivery services was significantly associated with women's greater likelihood of using facility-based delivery care after controlling for other covariates, while facilities' readiness to provide delivery services was also important in nonmetropolitan urban areas.

Conclusion: Increasing physical access to delivery care should become a high priority in rural Haiti. In urban areas, where delivery services are more available than in rural areas, improving quality of care at facilities could potentially lead to increased coverage of facility delivery.

\section{INTRODUCTION}

$\mathbf{H}$ aiti has the highest maternal mortality ratio in Latin America and the Caribbean, at an estimated 359 deaths per 100,000 live births. ${ }^{1}$ Every year,

a The Demographic and Health Surveys (DHS) Program, ICF, Rockville, MD, USA. Correspondence to Wenjuan Wang (wenjuan.wang@icf.com). thousands of women in Haiti die from causes that could be prevented by access to comprehensive and skilled obstetric care during pregnancy, childbirth, and the postpartum period. ${ }^{2,3}$ Use of maternal health services, especially facility delivery, remains low in Haiti. Only $36 \%$ of births take place in health facilities, according to the 2012 Haiti Demographic and Health Survey (DHS). ${ }^{4}$ Unless a woman delivers at a health facility, she is 
unlikely to have access to emergency obstetric care, which is considered the most important strategy for reducing maternal deaths., ${ }^{5,6}$ Increasing use of facility delivery is critical for reducing maternal mortality in Haiti.

An extensive body of literature exists on factors that influence facility delivery. ${ }^{7-11}$ The majority of studies have focused on the demand side-for example, the characteristics of women and their families. A few studies have looked at social contextual factors such as community norms, media access, and the level of local development. ${ }^{12-15}$ In Haiti, a few studies have found that facility delivery was associated with maternal and birth characteristics, household poverty, use of antenatal care services, and community exposure to mass media. ${ }^{16,17}$

The effect of the supply side-delivery care offered in health facilities-on facility delivery has received only limited attention., 811 One of the main reasons that research on the effects of service provision is limited is lack of suitable data. Supply-side data typically come from health facilities and need to be linked to data from population-based surveys in order to explore the relationship between the provision of services and women's use of facility-based delivery care.

With the availability of geographic data from both household surveys and health facility surveys, it has become possible to link population data and health facility data within a geographic information system (GIS). A few studies have linked DHS data and facility census data to assess how distance to the closest facility affects women's use of reproductive health services. ${ }^{18-20}$ For example, a study in Malawi and Zambia linking DHS clusters and health facilities (from a facility census) found that in both countries a longer straight-line distance from the DHS cluster to the closest facility offering emergency obstetric care significantly reduced the likelihood of facility delivery. ${ }^{19}$ Another study in Zambia with the same methodology but focusing on antenatal care found that distance to the closest facility had a significant effect on the content of care women received but had no effect on number of antenatal care visits or timing of the first visit. $^{18}$ In a rural setting in Ghana, Nesbitt and colleagues linked health facility census data and health and demographic surveillance data from about 600 villages and found a significant association between distance to the closest delivery facility and women's likelihood of delivering in a health facility. ${ }^{20}$
While these studies have contributed to establishing a geospatial methodology to assess the relationship between service provision and use, linking DHS clusters and closest facilities is subject to misclassification errors. Because DHS cluster coordinates are displaced before release of the data (to ensure respondent confidentiality is maintained), the closest facility identified based on the released geographic data may not be the nearest facility in reality. Skiles and colleagues indicated that, due to displacement of cluster coordinates, the distance to the closest facility can be misclassified for $34 \%$ to $43 \%$ of clusters. ${ }^{21}$ The displacement is an important limitation of the data, so instead of only looking at the closest facility our study measured the service environment (all available facilities) within a reasonable distance of the displaced cluster, thus representing the likely service environment of the real cluster location.

Because of Haiti's mountainous terrain, physical accessibility remains one of the biggest barriers to using health care. ${ }^{22,23}$ Gage and Guirlène Calixte linked women's reports on use of facility delivery care and community key informants' reports on health services and found that in rural Haiti the physical accessibility of health facilities was strongly associated with women's use of delivery care. ${ }^{23}$ Women's odds of being attended by a skilled birth attendant were positively associated with the presence of a health worker providing antenatal care in the neighborhood but negatively associated with living in a mountainous terrain and with distance from the nearest hospital. Ruktanonchai and colleagues found that in the 5 East Africa countries studied, geographic inaccessibility was an important predictor of use of maternal health care services, including skilled birth attendance. ${ }^{24}$ This furthers the argument that straight-line distance linkage between a facility and a DHS displaced cluster may cause misclassification in the Haiti context. Thus, the service environment approach is a better approximation of the likely access to health facilities for a woman.

While physical access is important, another key determinant of service utilization is the quality of care. Families may bypass the nearest health facility when quality is an issue. ${ }^{25-27}$ In examining the effect of the quality of care on use of services, some studies have looked at the quality of care from the user's perspective. ${ }^{15,28}$ While this perspective is indicative, it is subject to the respondents' level of knowledge about the services provided at health facilities, which may be biased or misinformed. Among the limited number of

\author{
Increasing use \\ of facility delivery \\ is critical for \\ reducing \\ maternal \\ mortality in Haiti.
}

\author{
Another key \\ determinant of \\ service utilization, \\ beyond physical \\ proximity, is the \\ quality of care of \\ services.
}


studies based on linked population data and facility data, a few have looked at the quality of service provision in health facilities. The study in Zambia by Kyei and colleagues measured the level of care using an index that combined several process and structural aspects of antenatal care provided at facilities. ${ }^{18}$ The level of service provision at the closest health facility was found to be significantly associated with the content of antenatal care received. In Nepal, when quality of care was measured solely in structural terms (e.g., infrastructure, availability of medicine, number of staff) a significant effect was also seen on the use of antenatal care and immunization services. ${ }^{29}$

Haiti has a hierarchical system of health care provision in which small facilities are located in villages or small communities and larger, betterequipped facilities are located in cities. Small or low-level facilities may not provide delivery serv-

We limited our analysis to women who had a live birth in the 5 years preceding the survey, with the final analysis sample comprising 4,921 women.

\section{This study used geographic data collected in the Haiti \\ Demographic and Health Survey (DHS) and the Service Provision Assessment (SPA) to link DHS clusters and SPA facilities.}

ices, however, and among those that provide these services there is little information on how prepared they are to provide good-quality delivery care and how their service preparedness affects utilization. The Haiti 2013 Service Provision Assessment (SPA) and 2012 DHS provide an opportunity to link data for health facilities and DHS clusters in order to explore the influence of service availability and readiness on use of delivery care in facilities.

\section{DATA AND METHODS}

\section{Data}

The Haiti DHS provides data on women's sociodemographic characteristics and their use of maternal health care services, including facilitybased delivery care. The Haiti SPA provides information on the availability of delivery care at health facilities and the readiness of facilities to provide good-quality services. This study used geographic data collected in both surveys to link DHS clusters and SPA facilities.

\section{Population Data}

The 2012 Haiti DHS is a population-based household survey that provides representative estimates for both urban and rural areas and for the 10 administrative departments of Haiti. The survey used a 2-stage cluster sampling design. At the first stage, 445 clusters were selected with probability proportional to their population size from a master national sample frame. At the second stage, a systematic sample of households was drawn in each of the selected clusters. All women ages 15-49 in the sampled households were eligible for individual interview, which collected data on their socio-demographic characteristics and use of health care services. A total number of 14,287 women were interviewed.

The Haiti DHS georeferenced the locations of the sampled clusters by using Global Positioning System (GPS) receivers to collect the coordinates of the center of the populated areas of the clusters. Prior to release of the geographic dataset, the cluster coordinates were verified and geographically displaced. ${ }^{30}$ Coordinates of urban clusters were displaced up to a maximum distance of 2 kilometers (km); average urban displacement was $0.8 \mathrm{~km}$. In rural areas, the displacement distance was up to $5 \mathrm{~km}$ with a further, randomly selected $1 \%$ of rural clusters displaced up to $10 \mathrm{~km}$; average rural displacement was $2.1 \mathrm{~km}$. These displaced GPS locations were used in our analysis.

Given that the outcome of interest was facility delivery, we limited the analysis to women who had a live birth in the 5 years preceding the survey $(\mathrm{N}=5,218)$ with a focus on their most recent birth. We excluded 234 women interviewed in 45 camp clusters that housed the population displaced by the 2010 earthquake because they were likely to have lived in a different area when they had their most recent birth. Thus, the health care environment where they had the birth was likely different from where they had been surveyed. Another 63 women were excluded from the analysis because they were from 8 clusters with missing georeferenced data. The final analysis sample consisted of 4,921 women from 392 clusters with GPS data available.

\section{Health Facility Data}

The 2013 Haiti SPA is a health facility census of 907 public and private facilities, from hospitals at the highest level to dispensaries at the lowest level. The SPA provides data on availability of key health services and readiness to provide these services. Data were collected using 4 types of survey instruments: the inventory questionnaire, health provider interviews, observation of consultations, and client exit interviews. For our analysis, facility data primarily came from the inventory questionnaire, which was administered to the facility manager or the most knowledgeable person for specific service areas. The inventory questionnaire collects data on the facility's infrastructure, supplies, medicines, staffing, trainings, and routine practices in providing general and specific services.

The SPA georeferenced the locations of the health facilities by using GPS receivers. Unlike the 
DHS GPS data for each cluster, facilities' GPS coordinates were not displaced. Data on 195 hospitals and health centers with a bed that offer delivery services were analyzed in this study. We excluded health centers without a bed and dispensaries from the analysis because they are not mandated to provide delivery care and are rarely used for delivery care. ${ }^{4,31}$ Figure 1 shows the location of the SPA facilities as well as the DHS (displaced) clusters that were included in the analysis.

Linked Data Between DHS Clusters and SPA Facilities Using publicly available GPS data for DHS (displaced) clusters and SPA facilities, we linked facilities to clusters to measure the health service environment of the clusters. We first created a distance matrix with the direct distance measurement from every DHS (displaced) cluster location to every health facility. The distances were then used to identify facilities within a 5-km buffer dis- tance from an urban cluster and a 10-km buffer distance from a rural cluster. These buffer sizes around the displaced location were chosen to ensure that facilities within the same distance around the real (non-displaced) location of the cluster were included within the buffer, given the displacement radius used for urban (maximum $2 \mathrm{~km}$ ) and rural locations (maximum $10 \mathrm{~km}$ ). We also believe that facilities within the chosen buffer distance reasonably represent the service environment where individuals seek health services, if not the exact facilities they visit. Lastly, data from all the facilities within the buffer distance were summarized for each cluster to measure the cluster's service environment.

Definitions of Key Measurements. The outcome variable was dichotomous, indicating whether a woman delivered her most recent birth at a health facility. We measured the service environment with 2 indicators: availability of facilities

\section{Data on 195 hospitals and health centers with a bed that offered delivery services were analyzed.}

FIGURE 1. Geographic Distribution of 2012 DHS (Displaced) Clusters and 2013 SPA Facilities Included in the Analysis

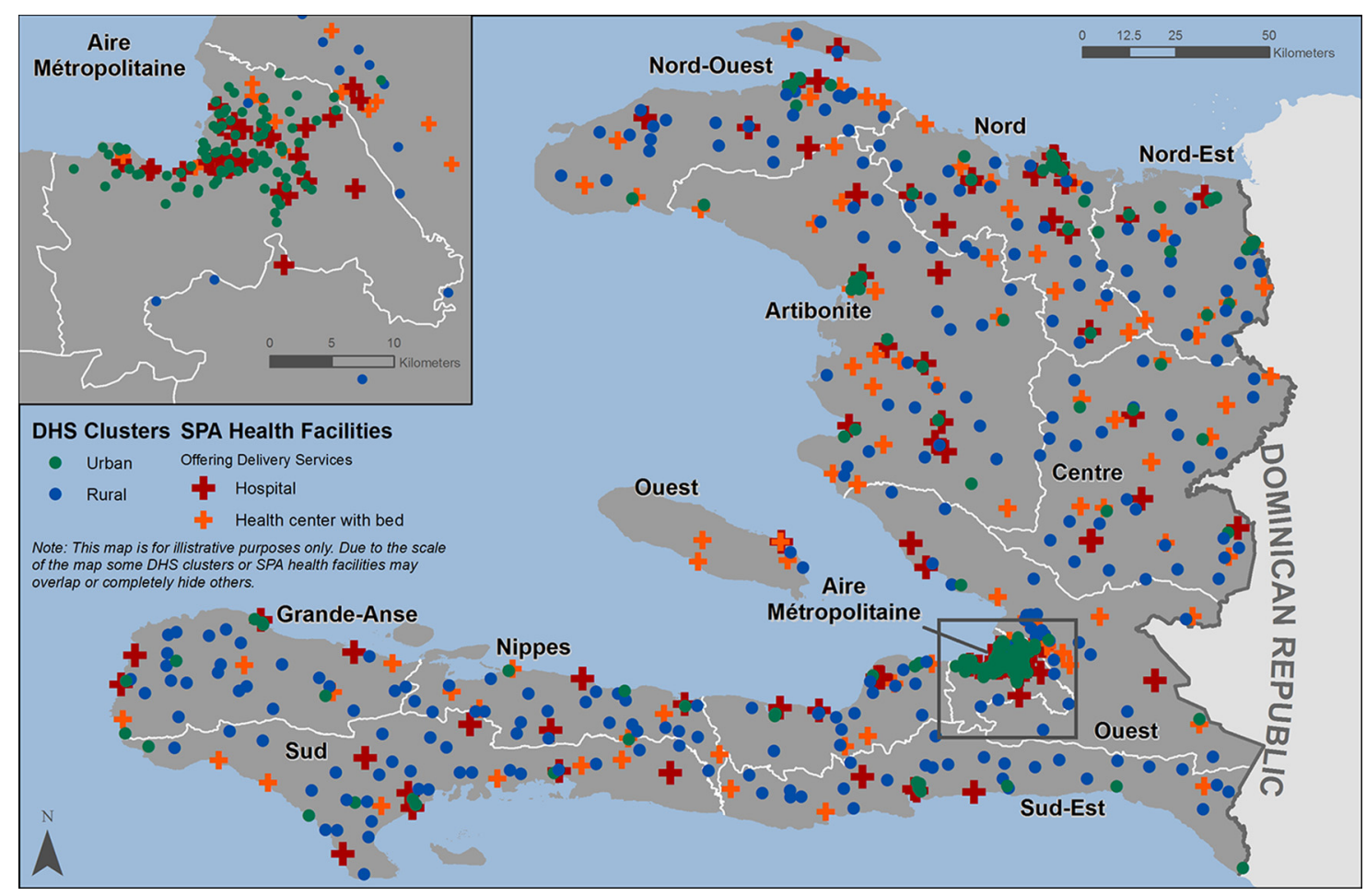

Abbreviations: DHS, Demographic and Health Survey; SPA, Service Provision Assessment. 


We measured a
facility's readiness
to provide good-
quality delivery
care by a
readiness score
based on
37 indicators
defined by WHO.

We categorized
clusters into
groups with
3 levels of
availability of
delivery services:
low, medium, or
high.

We measured
facilities'
readiness to
provide delivery
services by the
median readiness
score of all the
facilities within the
buffer and divided
clusters into low-,
medium-, or high-
level readiness
groups based on
the score terciles.

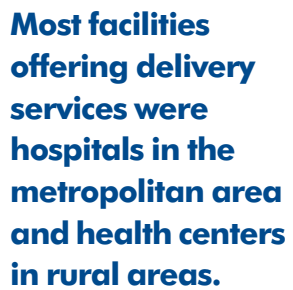

offering delivery care and facilities' readiness to provide good-quality delivery care. Both were measured at the DHS cluster level and derived from the facility-level data after linking facilities to DHS clusters.

At the facility level, we measured availability using the SPA definition of facilities offering delivery care, which the SPA obtained by asking the facility manager if the facility provides delivery services. We measured facility's readiness to provide good-quality delivery care by a readiness score created with principal component analysis based on a set of service readiness indicators defined by the World Health Organization. ${ }^{32}$ For each indicator-according to whether the facility met the criteria for availability-facilities were assigned a binary variable: $1=$ available, $0=$ unavailable. A total of 37 readiness indicators were constructed; their definitions are presented in Supplementary Table 1. The readiness score was computed based on the first component resulting from the principal component analysis, which explained the largest proportion of the total variance. The readiness score is a relative summary indicator of how ready a health facility is to provide good-quality delivery services. A higher score represents better readiness and a lower score represents poorer readiness compared with other facilities. Availability of delivery service and readiness indicators were then summarized to the DHS cluster level, as described below.

To measure availability of delivery services, we counted the number of facilities offering delivery services within the specified buffer of each DHS cluster. We categorized clusters into groups with 3 levels of availability:

1. Low availability: no facility with delivery services within the buffer distance

2. Medium availability: 1 facility with delivery services within the buffer distance

3. High availability: 2 or more facilities with delivery services within the buffer distance

Facilities' readiness to provide delivery care was measured by the median readiness score of all the facilities within the buffer. Given that the readiness score is a relative measurement, we divided the clusters into low-, medium-, and highlevel readiness groups based on the score terciles at the cluster level. Clusters with a median score (median score of all facilities within the buffer) falling in the top 33\% were considered in the high-level readiness group; those with a median score in the bottom $33 \%$ were categorized into the low-level readiness group; and the rest were put in the medium-level readiness group.

\section{Statistical Analysis}

The analysis was stratified by urban and rural residence because of differences between urban and rural areas in the health service environment and women's health care-seeking behaviors. We further separated the Port-au-Prince metropolitan area (comprising the capital city Port-au-Prince and the urban zones of the Ouest region) from other urban areas because of the substantial differences in the density and types of health facilities.

Random-intercept logistic regressions were used for the multivariable analysis. DHS data follow a hierarchical structure-that is, individuals are nested within households and households are nested within clusters. Respondents who live in the same household or cluster may not be independent of one another. Moreover, the outcome variable is at the individual level but the key exploratory variables-level of service availability and readiness of facilities to provide delivery services-are at the cluster level. A multilevel analysis approach is more appropriate to allow for simultaneous investigation of the effect of the group-level and individual-level predictors on individual-level outcomes. ${ }^{33}$ Therefore, we applied multilevel (individual- and cluster-level) random intercept logistic models to investigate how the service environment measures affect women's use of facility-based delivery care. The household level was omitted since there were few women who had a child in the 5 years preceding the survey living in the same household.

Other variables adjusted for included women's age at birth, birth order, mother's education, household wealth quintile, number of antenatal care visits, and region (department), all of which have been found to be associated with facility delivery. ${ }^{12,34}$

\section{RESULTS}

\section{Sample Characteristics}

For health facilities, we analyzed data on hospitals and health centers with a bed that provide delivery services. The distribution of these facilities in each residence location by facility background characteristics is presented in Supplementary Table 2. Overall, hospitals and health centers each accounted for half the facilities; but in the metropolitan area most facilities offering delivery services were hospitals, while in rural areas most were health centers. With regard to the managing 
authority, more than half of the facilities in the metropolitan area were private for-profit, while government health facilities were more common in other urban areas and rural areas. Facility distribution also varied across regions in rural and other urban areas. In rural areas, some regions, such as the Ouest region, had many health facilities, while some, such as Sud-Est and Grand-Anse, had only a few.

For use of facility-based delivery services, data were based on 4,921 women who had a live birth in the 5 years preceding the DHS survey (including 2,878 women in rural areas, 1,214 in the metropolitan area, and 829 in other urban areas). Supplementary Table 3 provides the distribution of these women by background characteristics and receipt of antenatal care for the most recent birth. In all 3 location categories, a majority of the women reported having their most recent birth between ages $20-34$, while $14 \%$ to $15 \%$ were under age 20 when they had their most recent birth. Most women in the metropolitan and other urban areas had 3 children or fewer, with about $40 \%$ having the most recent birth as their first birth. In contrast, in rural areas a higher percentage of women $(38 \%)$ had 4 children or more. In the metropolitan and other urban areas, most women reported having a primary education and close to $60 \%$ had secondary or higher education. In contrast, in rural areas only $25 \%$ of women had secondary or higher education, while $27 \%$ had no education. In the metropolitan and other urban areas, women in the study were pri- marily in the upper 3 wealth quintiles, while in rural areas $71 \%$ of women were in the poorest 2 quintiles. As for women's receipt of antenatal care during pregnancy for their most recent birth, more than three-quarters of women in the metropolitan and other urban areas had 4 or more antenatal care visits compared with less than two-thirds $(60 \%)$ of rural women.

\section{Availability of Delivery Services}

Availability of facilities offering delivery services varied by location; there was a greater number of facilities within the buffer of clusters in the metropolitan area than in other urban areas and rural areas. The number of facilities with delivery services within the buffer distances from the DHS clusters (i.e., within $5 \mathrm{~km}$ for the metropolitan and other urban areas and within $10 \mathrm{~km}$ for rural areas) ranged from $0-28$ in the metropolitan area, from 0-6 in other urban areas, and from 0-7 in rural areas. Figure 2 presents the percentage distribution of women by levels of availability to delivery services. Proximity to a facility with delivery services was nearly universal in the metropolitan area; $99 \%$ of women lived in an area with a high level of availability of delivery services ( 2 or more facilities within $5 \mathrm{~km}$ from their clusters). In contrast, $12 \%$ of women in other urban areas and $18 \%$ of rural women lived in areas with low availability-that is, no facility offering delivery services within $5 \mathrm{~km}$ for women in other urban areas and $10 \mathrm{~km}$ for women in rural areas.

FIGURE 2. Percent Distribution of Women by Level of Availability of Facilities With Delivery Services Within the Buffer Distance, ${ }^{a}$ Haiti, 2012-2013

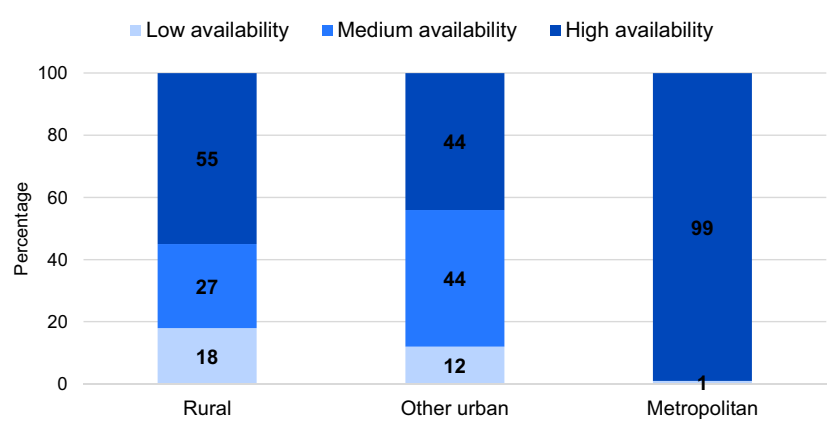

a Low availability=no facility with delivery services within the buffer distance; medium availability= 1 facility with delivery services within the buffer; high availability=2 or more facilities with delivery services within the buffer. The buffer distance was $5 \mathrm{~km}$ for urban clusters and $10 \mathrm{~km}$ for rural clusters.

\section{Proximity to a facility with delivery services was nearly universal in the metropolitan area, whereas $12 \%$ of women in other urban areas and $18 \%$ of rural women lived in areas with no such facility.}




\section{Availability of comprehensive obstetric care items was low across the board, but especially in facilities in rural areas.}

\section{In both urban and rural areas, availability of delivery services was significantly associated with women's use of facility delivery care.}

\section{Facilities' Readiness to Provide Delivery Services}

Readiness to provide delivery services among facilities that offered delivery services was measured by the availability of a number of basic and comprehensive obstetric care services and items available at the facility on the day of the survey. Table 1, which presents the availability of these 37 services and supplies, shows wide variation by the facility's location. Facilities in the metropolitan and other urban areas had many of the items while those in rural areas had few. Some items were commonly available across locations, such as a delivery bed, gloves, injectable antibiotics, and parenteral administration of oxytocic drug, while other items were rare, such as manual vacuum extractor and guidelines for integrated management of pregnancy and childbirth. Overall, the availability of comprehensive obstetric care items was low, especially at facilities in rural areas.

As discussed earlier, to assess the level of service readiness at facilities that the DHS cluster was linked to, an overall readiness score was generated for each cluster based on the median score of all facilities within the buffer. The clusters were divided into low-, medium-, and high-readiness groups based on the readiness score terciles at the cluster level. Figure 3 shows that the distribution of readiness scores differed by residence area. In the metropolitan area, the cluster readiness scores were more homogenous, while the interquartile range was wider in other urban and rural clusters. The readiness score distribution in other urban areas was highly skewed to high readiness scores, indicating that some facilities had much better readiness than others. A few outlier facilities with markedly different (better or worse) readiness compared with the rest of facilities were also observed in each residence area.

\section{Use of Facility Delivery Services by Level of Availability and Readiness of Delivery Services}

Overall, 39\% of women delivered their most recent birth at a health facility and $61 \%$ delivered at home. Delivery at a health facility was far more common in the metropolitan and other urban areas $(60 \%$ and $59 \%$, respectively) compared with rural areas $(24 \%)$.

Figure 4 highlights the bivariate association between availability of delivery services and the percentage of women who delivered their most recent birth at a health facility. Since nearly all women in the metropolitan area lived in clusters with 2 or more facilities within $5 \mathrm{~km}$, the effect of availability of delivery services was assessed only for other urban and rural areas. In both types of area, use of facility delivery was significantly associated with levels of availability of facilities offering delivery services. In rural areas, facility delivery coverage increased incrementally with the level of availability. In rural clusters without a facility with delivery services within $10 \mathrm{~km}$, only $8 \%$ of women delivered the most recent birth in a facility compared with $19 \%$ of women in clusters with access to 1 such facility, and $32 \%$ of those with 2 or more such facilities. Service availability was also associated with use in other urban areas but the difference was less remarkable between medium and high availability.

Figure 5 indicates that use of facility delivery services was also positively associated with the level of readiness at the facilities within the buffer distance in the metropolitan and other urban areas. In other urban clusters, $45 \%$ of women delivered in a facility with low level of readiness compared with $63 \%$ of women for facilities with a medium level of readiness and $71 \%$ of women for facilities with a high level of readiness. In rural areas, however, there was little association between facilities' level of readiness and women's use of facility delivery services.

\section{Results of Multivariable Analysis}

Using random-intercept logistic models, we assessed how women's use of facility delivery was associated with availability of delivery services within the buffer and service readiness of the facilities. We first ran models for nonmetropolitan urban areas and rural areas to examine the effect of availability. This model was not run for the metropolitan area since almost all metropolitan women had a high level of availability to delivery services. We then ran models to examine the effect of service readiness for all 3 locations after controlling for availability and other covariates. Since readiness is only available for clusters with at least 1 facility within the buffer distance, women in clusters with no facility were dropped from this model.

Table 2 presents odds ratios (ORs) and 95\% confidence intervals (CIs) from the regression models of facility delivery on the availability indicator, as well as for the covariates adjusted for in the models. In both urban and rural areas, availability of delivery services was significantly associated with women's use of facility delivery care after controlling for other covariates. In rural 
TABLE 1. Percentage of Facilities Offering Delivery Services With Availability of Basic and Comprehensive Obstetric Care Services and Items, by Residence, Haiti, 2013

\begin{tabular}{|c|c|c|c|c|}
\hline & Rural \% & Other Urban \% & Metropolitan \% & Total \% \\
\hline \multicolumn{5}{|l|}{ Basic obstetric care indicators } \\
\hline Parenteral administration of antibiotics & 68.9 & 78.0 & 94.9 & 77.9 \\
\hline Parenteral administration of oxytocic drug & 86.5 & 95.1 & 94.9 & 91.8 \\
\hline Parenteral administration of anticonvulsants & 40.5 & 67.1 & 48.7 & 53.3 \\
\hline Assisted vaginal delivery ${ }^{a}$ & 90.5 & 92.7 & 89.7 & 91.3 \\
\hline Manual removal of placenta & 58.1 & 79.3 & 59.0 & 67.2 \\
\hline Manual removal of retained products & 48.6 & 75.6 & 64.1 & 63.1 \\
\hline Neonatal resuscitation & 51.4 & 72.0 & 53.8 & 60.5 \\
\hline Guidelines for IMPAC & 21.6 & 31.7 & 12.8 & 24.1 \\
\hline Staff trained in IMPAC & 48.6 & 53.7 & 46.2 & 50.3 \\
\hline Emergency transportaion & 32.4 & 36.6 & 35.9 & 34.9 \\
\hline Sterilization equipment & 75.7 & 84.1 & 87.2 & 81.5 \\
\hline Examination light & 43.2 & 43.9 & 59.0 & 46.7 \\
\hline Delivery pack & 87.8 & 92.7 & 87.2 & 89.7 \\
\hline Suction apparatus & 93.2 & 98.8 & 79.5 & 92.8 \\
\hline Manual vacuum extractor & 16.2 & 19.5 & 20.5 & 18.5 \\
\hline Vacuum aspirator or D\&C kit & 20.3 & 35.4 & 30.8 & 28.7 \\
\hline Newborn bag and mask & 51.4 & 62.2 & 59.0 & 57.4 \\
\hline Delivery bed & 97.3 & 100.0 & 94.9 & 97.9 \\
\hline Partograph & 32.4 & 41.5 & 33.3 & 36.4 \\
\hline Gloves & 93.2 & 93.9 & 89.7 & 92.8 \\
\hline Antibiotic eye ointment for newborns & 66.2 & 84.1 & 66.7 & 73.8 \\
\hline Injectable uterotonic & 62.2 & 74.4 & 64.1 & 67.7 \\
\hline Injectable antibiotics & 89.2 & 92.7 & 94.9 & 91.8 \\
\hline Injectable magnesium sulphate & 60.8 & 78.0 & 74.4 & 70.8 \\
\hline Skin disinfectant & 60.8 & 81.7 & 61.5 & 69.7 \\
\hline Intravenous solution with infusion set & 74.3 & 84.1 & 82.1 & 80.0 \\
\hline Regular reviews of maternal or newborn deaths & 25.7 & 32.9 & 48.7 & 33.3 \\
\hline \multicolumn{5}{|l|}{ Comprehensive obstetric care indicators } \\
\hline Cesarean delivery services & 23.0 & 52.4 & 71.8 & 45.1 \\
\hline Blood transfusion & 23.0 & 50.0 & 61.5 & 42.1 \\
\hline
\end{tabular}


TABLE 1. Continued

\begin{tabular}{|c|c|c|c|c|}
\hline & Rural \% & Other Urban \% & Metropolitan \% & Total \% \\
\hline Guidelines for CEmOC adapted for Haiti & 6.8 & 15.9 & 7.7 & 10.8 \\
\hline Staff member providing delivery trained in $\mathrm{CEmOC}$ & 44.6 & 45.1 & 38.5 & 43.6 \\
\hline Anesthesia equipment & 10.8 & 15.9 & 25.6 & 15.9 \\
\hline Incubator & 10.8 & 23.2 & 23.1 & 18.5 \\
\hline Blood typing & 17.6 & 42.7 & 56.4 & 35.9 \\
\hline Cross matching test & 4.1 & 3.7 & 10.3 & 5.1 \\
\hline Blood supply sufficiency & 12.2 & 19.5 & 20.5 & 16.9 \\
\hline Blood supply safety & 16.2 & 37.8 & 53.8 & 32.8 \\
\hline Total number of facilities & 74 & 82 & 39 & 195 \\
\hline
\end{tabular}

FIGURE 3. Distribution of Cluster-Level Median Readiness Scores Among Facilities Offering Delivery Services, by Residence, Haiti, 2012-2013

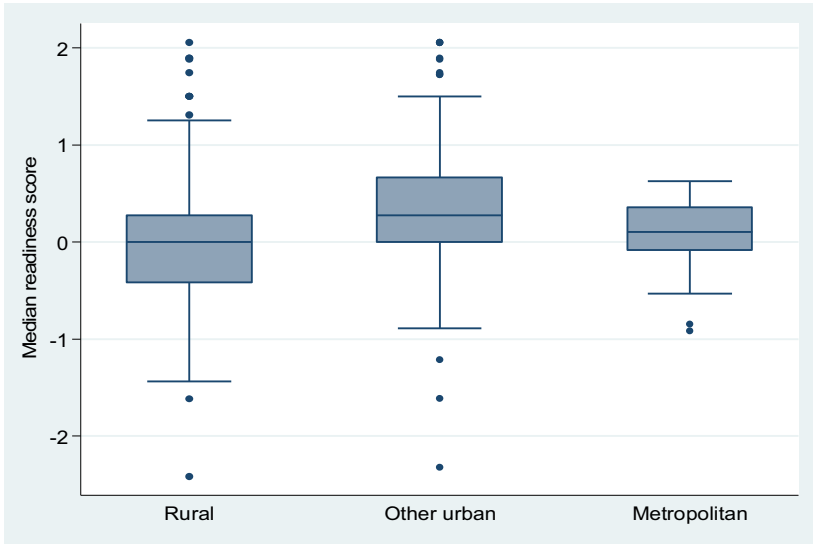

areas, women in clusters with medium-level availability to delivery services had 1.9 times higher odds of going to a facility for delivery (CI, 1.14 to $3.13 ; P<.05$ ) and women in clusters with high availability had 3.6 times higher odds (CI, 2.23 to 5.66; $P<.001$ ) compared with women with low availability. Similarly, in other urban areas higher availability of delivery services was also significantly associated with a greater likelihood to deliver in a facility. Although the odds ratio appears lower for high availability (OR, $2.64 ; 95 \%$ CI, 1.13 to $6.19 ; P<.05)$ than medium availability (OR, 3.83; 95\% CI, 1.57 to 9.31; $P<.01)$, the difference was not statistically significant (results not shown).

As expected, maternal age at birth, the number of antenatal care visits, and household wealth were positively and significantly associated with 
FIGURE 4. Percentage of Women Who Delivered at a Facility by Availability of Facilities With Delivery Services Within the Buffer Distance, ${ }^{a}$ Haiti, 2012-2013

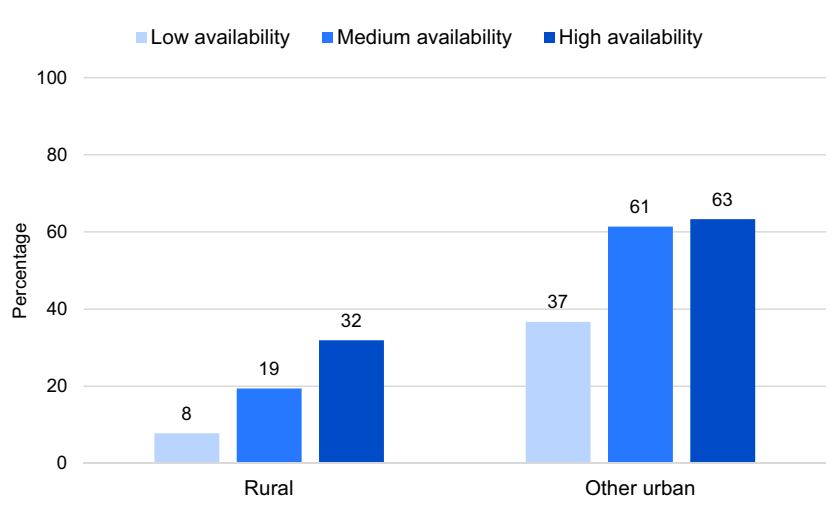

a Low availability=no facility with delivery services within the buffer distance; medium availability= 1 facility with delivery services within the buffer; high availability=2 or more facilities with delivery services within the buffer. The buffer distance was $5 \mathrm{~km}$ for urban clusters and $10 \mathrm{~km}$ for rural clusters.

FIGURE 5. Percentage of Women Who Delivered at a Facility by Level of Readiness ${ }^{a}$ at Facilities With Delivery Services Within the Buffer Distance, ${ }^{\text {Haiti, } 2012-2013}$

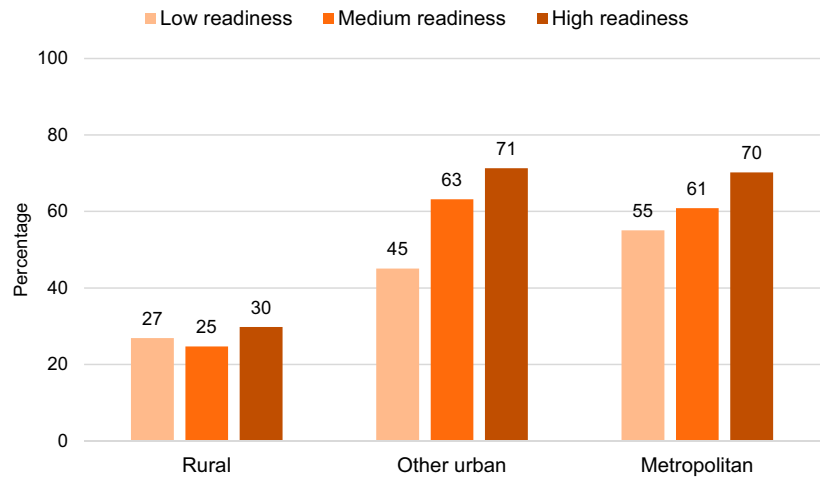

a Low-, medium-, and high-level readiness=median readiness score of the facilities within the buffer falling in the bottom 33.3\%, middle $33.3 \%$, and top 33.3\%, respectively. The buffer distance was $5 \mathrm{~km}$ for urban clusters and $10 \mathrm{~km}$ for rural clusters.

b Women living in clusters with no health facility offering delivery services within the buffer distance were excluded from this figure since the readiness score was applied only to clusters with 1 or more health facilities.

women's use of delivery care in a health facility, while the child's birth order was negatively associated.

Table 3 shows the results of the regression models of facility delivery on service readiness and availability after controlling for women's characteristics and antenatal care variables. Service readiness Service readiness was significantly associated was significantly with facility delivery only in urban areas (other associated with than the metropolitan area). Women in clusters facility delivery with a high level of service readiness had 2.7 times only in urban higher odds of delivery in a health facility areas. 
TABLE 2. Multivariable Model Results for the Regressions of Facility Delivery on Availability of Facilities With Delivery Services Within the Buffer, Controlling for Other Variables, Haiti, 2012-2013

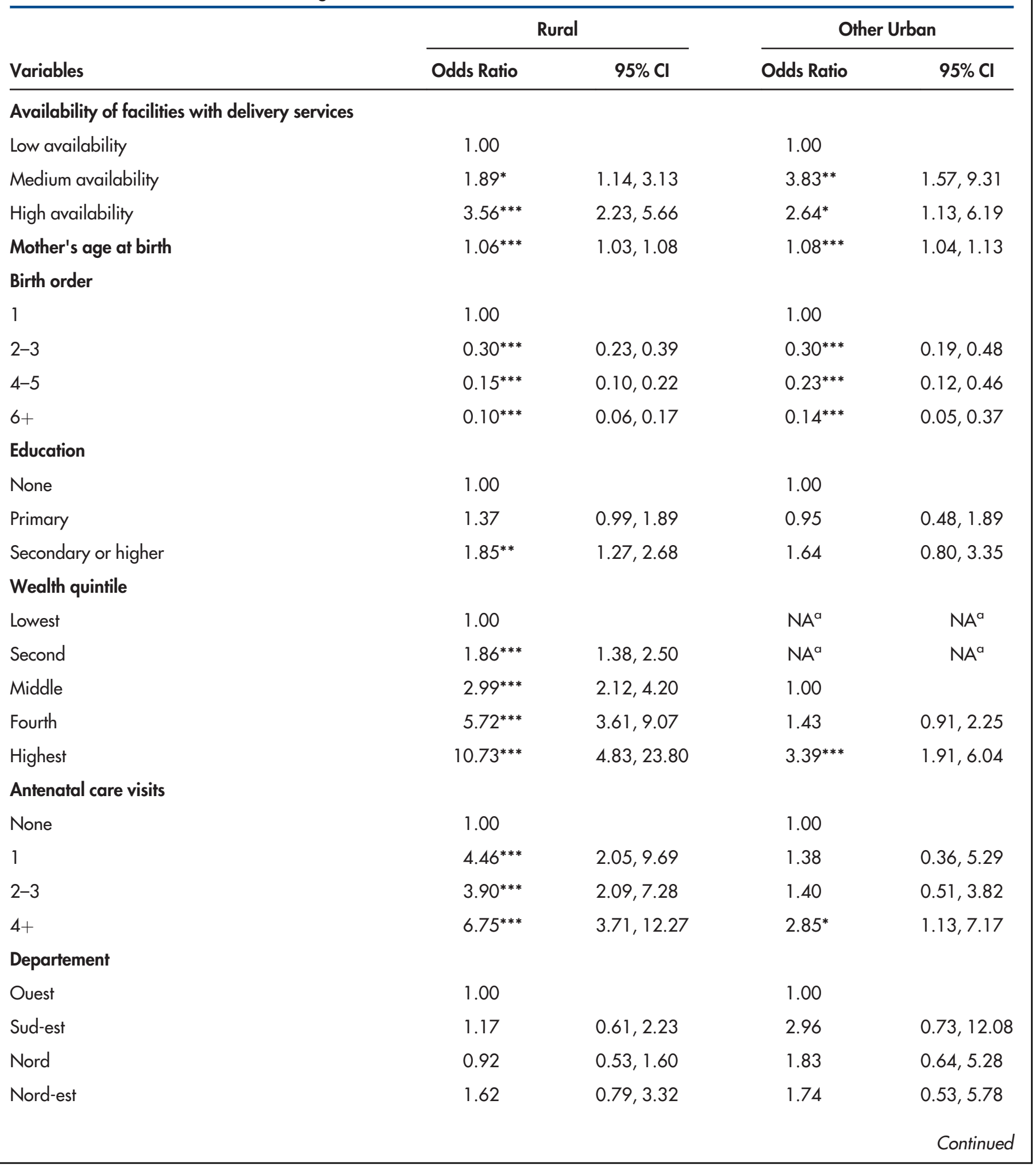


TABLE 2. Continued

\begin{tabular}{|c|c|c|c|c|}
\hline \multirow[b]{2}{*}{ Variables } & \multicolumn{2}{|c|}{ Rural } & \multicolumn{2}{|c|}{ Other Urban } \\
\hline & Odds Ratio & $95 \% \mathrm{Cl}$ & Odds Ratio & $95 \% \mathrm{Cl}$ \\
\hline Artibonite & 1.17 & $0.76,1.78$ & 1.37 & $0.49,3.86$ \\
\hline Centre & 1.64 & $0.97,2.78$ & 1.42 & $0.39,5.20$ \\
\hline Sud & 1.56 & $0.94,2.59$ & 1.90 & $0.52,6.88$ \\
\hline Grand'anse & 1.21 & $0.57,2.57$ & 0.68 & $0.17,2.73$ \\
\hline Nord-ovest & 1.04 & $0.56,1.93$ & 0.67 & $0.19,2.39$ \\
\hline Nippes & 1.35 & $0.67,2.70$ & 1.71 & $0.26,11.14$ \\
\hline Number of clusters & 241 & & 85 & \\
\hline Observations & 2,878 & & 829 & \\
\hline
\end{tabular}

compared with women in clusters with low readiness ( $95 \% \mathrm{CI}, 1.34$ to $5.60 ; P<.01)$. The difference in facility delivery between women in clusters with low readiness and those with medium readiness was not significant after controlling for other covariates. Similar to the findings from the models on availability, in other urban areas there was no significant difference in coverage of facility delivery between clusters with medium availability and clusters with high availability of delivery services. In rural areas, after controlling for service readiness and other variables, having high availability of delivery services-that is, having 2 or more facilities within the buffer distance-was associated with significantly greater odds of delivery in a health facility compared with medium availability-that is, having only 1 facility with delivery services within the buffer distance (OR, $1.94 ; 95 \%$ CI, 1.38 to $2.72 ; P<.001)$.

\section{DISCUSSION}

The availability of the recent DHS and SPA surveys in Haiti, both with GPS data, enabled analysis linking women's use of facility delivery care with their physical access to delivery care and facilities' readiness to provide the care. This analysis showed that women in rural areas of Haiti have very limited physical access to obstetric care. Almost
1 in 5 women in rural areas lives in a place where there is no facility that provides delivery services within a $10 \mathrm{~km}$ distance. It should be noted that the $10 \mathrm{~km}$ distance is a straight-line measurement. The actual travel distance could be longer because of travel paths, road networks, and the mountainous terrain. In rural areas, physical access to health care can be further constrained by poor road conditions and lack of transportation.

The regression results highlight the importance of service availability to the use of facility delivery in rural areas. Living reasonably near a facility that provides delivery services is significantly associated with greater probability of women delivering at a health facility. Physical access as an important barrier to service use in rural Haiti was also demonstrated in previous research that measured access with the distance to the nearest facility reported by key community informants. ${ }^{23}$ Our finding also agrees with previous research that having physical access to services is a strong determinant of use of delivery care. ${ }^{28,34-36}$

Quality of care-measured in our study by analyzing how prepared the facility is to provide the needed services-is important to the use of health services. Our results show that hospitals and health centers with a bed are not well prepared to provide delivery services. A large number

\author{
Living reasonably \\ near a facility that \\ provides delivery \\ services is \\ significantly \\ associated with \\ greater \\ probability of \\ women delivering \\ at a health facility. \\ Women in rural
areas of Haiti
have very limited
physical access to
obstetric care.
}


TABLE 3. Multivariable Model Results for the Regressions of Facility Delivery on Service Readiness of Facilities With Delivery Services, Controlling for Availability of Services and Other Variables, Haiti, 2012-2013

\begin{tabular}{|c|c|c|c|c|c|c|}
\hline \multirow[b]{2}{*}{ Variables } & \multicolumn{2}{|c|}{ Rural } & \multicolumn{2}{|c|}{ Other Urban } & \multicolumn{2}{|c|}{ Metropolitan } \\
\hline & Odds Ratio & $95 \% \mathrm{Cl}$ & Odds Ratio & $95 \% \mathrm{Cl}$ & Odds Ratio & $95 \% \mathrm{Cl}$ \\
\hline \multicolumn{7}{|c|}{ Facilities' readiness to provide delivery services ${ }^{a}$} \\
\hline Low readiness & 1.00 & & 1.00 & & 1.00 & \\
\hline Medium readiness & 1.43 & $0.93,2.18$ & 1.74 & $0.71,4.26$ & 1.02 & $0.74,1.41$ \\
\hline High readiness & 1.33 & $0.95,1.85$ & $2.74^{* *}$ & $1.34,5.60$ & 1.41 & $0.96,2.05$ \\
\hline \multicolumn{7}{|c|}{ Availability of facilities with delivery services ${ }^{b}$} \\
\hline Medium availability & 1.00 & & 1.00 & & NA & \\
\hline High availability & $1.94^{* * *}$ & $1.38,2.72$ & 0.72 & $0.37,1.41$ & NA & \\
\hline Mother's age at birth & $1.05^{* * *}$ & $1.03,1.08$ & $1.09 * * *$ & $1.05,1.14$ & 1.023 & $0.99,1.05$ \\
\hline \multicolumn{7}{|l|}{ Birth order } \\
\hline 1 & 1.00 & & 1.00 & & 1.00 & \\
\hline $2-3$ & $0.31^{* * *}$ & $0.23,0.42$ & $0.28^{* * *}$ & $0.17,0.47$ & $0.61^{* *}$ & $0.43,0.88$ \\
\hline $4-5$ & $0.15^{* * *}$ & $0.10,0.24$ & $0.22^{* * *}$ & $0.10,0.48$ & $0.50^{*}$ & $0.29,0.86$ \\
\hline $6+$ & $0.12^{* * *}$ & $0.07,0.20$ & $0.17^{* *}$ & $0.06,0.49$ & $0.42^{*}$ & $0.20,0.91$ \\
\hline \multicolumn{7}{|l|}{ Education } \\
\hline None & 1.00 & & 1.00 & & 1.00 & \\
\hline Primary & $1.47^{*}$ & $1.04,2.08$ & 0.91 & $0.42,1.96$ & $3.23^{* * *}$ & $1.71,6.12$ \\
\hline Secondary or higher & $2.06^{* * *}$ & $1.39,3.06$ & 1.91 & $0.86,4.24$ & $5.01^{* * *}$ & $2.63,9.55$ \\
\hline \multicolumn{7}{|l|}{ Wealth quintile ${ }^{c}$} \\
\hline Lowest & 1.00 & & $N A^{c}$ & $N A^{c}$ & $N A^{c}$ & $N A^{c}$ \\
\hline Second & $1.89^{* * *}$ & $1.37,2.61$ & $N A^{c}$ & $N A^{c}$ & $N A^{c}$ & $N A^{c}$ \\
\hline Middle & $3.04^{* * *}$ & $2.11,4.38$ & 1.00 & & 1.00 & \\
\hline Fourth & $5.72^{* * *}$ & $3.56,9.21$ & 1.47 & $0.87,2.46$ & 1.05 & $0.68,1.63$ \\
\hline Highest & $11.14^{* * *}$ & $4.95,25.07$ & $2.82^{* *}$ & $1.49,5.35$ & $2.56^{* * *}$ & $1.55,4.20$ \\
\hline \multicolumn{7}{|l|}{ Antenatal care visits } \\
\hline None & 1.00 & & 1.00 & & 1.00 & \\
\hline 1 & $4.75^{* * *}$ & $2.05,10.99$ & 2.02 & $0.47,8.73$ & $3.60^{*}$ & $1.25,10.32$ \\
\hline $2-3$ & $4.17^{* * *}$ & $2.13,8.17$ & 2.16 & $0.69,6.76$ & $2.20^{*}$ & $1.12,4.32$ \\
\hline $4+$ & $7.24^{* * *}$ & $3.81,13.79$ & $3.88^{*}$ & $1.36,11.05$ & $3.95^{* * *}$ & $2.17,7.18$ \\
\hline & & & & & & Continued \\
\hline
\end{tabular}


TABLE 3. Continued

\begin{tabular}{|c|c|c|c|c|c|c|}
\hline \multirow[b]{2}{*}{ Variables } & \multicolumn{2}{|c|}{ Rural } & \multicolumn{2}{|c|}{ Other Urban } & \multicolumn{2}{|c|}{ Metropolitan } \\
\hline & Odds Ratio & $95 \% \mathrm{Cl}$ & Odds Ratio & $95 \% \mathrm{Cl}$ & Odds Ratio & $95 \% \mathrm{Cl}$ \\
\hline \multicolumn{7}{|l|}{ Departement } \\
\hline Ouest & 1.00 & & 1.00 & & & \\
\hline Sud-est & 0.98 & $0.47,2.03$ & 1.00 & $0.11,9.03$ & & \\
\hline Nord & 0.89 & $0.50,1.57$ & 2.00 & $0.57,6.98$ & & \\
\hline Nord-est & 1.63 & $0.79,3.39$ & 2.37 & $0.55,10.15$ & & \\
\hline Artibonite & 1.19 & $0.75,1.88$ & 1.60 & $0.46,5.62$ & & \\
\hline Centre & 1.64 & $0.92,2.92$ & 1.92 & $0.46,7.94$ & & \\
\hline Sud & 1.62 & $0.95,2.76$ & 2.46 & $0.48,12.64$ & & \\
\hline Grand'anse & 0.97 & $0.40,2.34$ & 0.58 & $0.12,2.79$ & & \\
\hline Nord-ouest & 1.02 & $0.54,1.92$ & 0.65 & $0.16,2.68$ & & \\
\hline Nippes & 1.36 & $0.66,2.81$ & 2.67 & $0.31,22.83$ & & \\
\hline Number of clusters & \multicolumn{2}{|l|}{201} & \multicolumn{2}{|l|}{69} & \multicolumn{2}{|l|}{59} \\
\hline Observations & \multicolumn{2}{|l|}{2,571} & \multicolumn{2}{|l|}{802} & \multicolumn{2}{|l|}{650} \\
\hline \multicolumn{7}{|c|}{$\begin{array}{l}\text { a Regressions excluded women living in clusters with no health facility offering delivery services within the buffer since the readiness score is applied only to } \\
\text { clusters with } 1 \text { or more health facilities. } \\
\text { b Since the analysis excluded women living in clusters with no health facility offering delivery services within the buffer (i.e., the low availability group), the } \\
\text { medium availability group was set as the reference. } \\
\text { 'In the metropolitan and other urban areas, no women were in the first wealth quintile and very few women were in the second wealth quintile; they were } \\
\text { combined into the third quintile. } \\
{ }_{\star \star * * P<.001 ; ~}^{* \star * P<.01 ;}{ }^{\star} P<.05 \text {. }\end{array}$} \\
\hline
\end{tabular}

of facilities lack essential equipment and supplies for routine delivery care, and most have limited capacity to provide emergency obstetric care. Only about one-third of facilities have functional emergency transportation, and the availability of Cesarean delivery services is limited, especially in rural areas. Delivery in a health facility itself cannot reduce maternal mortality unless women are assisted by a skilled birth attendant capable of managing common life-threatening obstetric complications. ${ }^{38}$ In Haiti, however, less than half of the facilities have staff who received training in comprehensive emergency obstetric care (CEmOC) during the last 2 years. Guidelines for CEmOC were not available in service areas at most facilities. All of these limitations increase the risk of death for mother and newborn when an obstetrical emergency occurs.

Despite the poor readiness of health facilities to provide delivery services, service quality appears to play an important role predicting facility delivery in urban areas, where delivery services are more available, compared with rural areas. Urban areas have more facilities, more accessible transportation, and more financial resources, and thus women may be able to choose to deliver at facilities offering a better quality of care. In rural areas, however, especially in mountainous areas, there is less availability of facilities and thus having access to at least 1 health facility seems more important to the use of delivery care than the facility's level of readiness to provide the services.

We did not find an association between service readiness and use of facility delivery in the metropolitan area. This could be because a $5 \mathrm{~km}$ buffer may not be appropriate for defining the service environment for clusters in the metropolitan area. Because of a high density of health facilities and DHS clusters in the metropolitan area, using a $5 \mathrm{~km}$ buffer may result in adjacent clusters

\section{Hospitals and health centers with a bed in Haiti are not well prepared to provide delivery services.}




Linking women's
use of health
services to the
service
environment
within a
reasonable
distance from DHS
clusters is less
prone to misclas-
sification errors
(resulting from the
displacement of
DHS clusters) than
linking to the
closet facility.

linking to more or less the same group of facilities; therefore, there is limited variation across metropolitan clusters in terms of the service environment. This is indicated by less variation in readiness among clusters in the metropolitan area compared with clusters in rural and other urban areas. Moreover, people in metropolitan areas are likely to have more transportation options available and better accessibility to health services that are geographically further from their home.

\section{Strengths and Limitations}

As discussed, because of methodological constraints in linking population data and health service data, most previous studies have been limited to measuring service provision from the client's perspective. Several recent studies have taken advantage of geographic data to associate health facilities and DHS clusters. These studies focused primarily on distance to the closest facility or the service available in the closest facility; however, this approach can be problematic because DHS cluster locations have been displaced. Our study improved on this methodology; instead of looking at a single facility (the closest facility) — where estimates may be subject to misclassification errorswe measured the effect of the service environment within a reasonable distance. In addition to the methodological improvement of measuring the service environment, some of the other strengths of our study lie in the use of facility census data and nationally representative household data, measuring service readiness with a wide range of items that the World Health Organization has identified as essential for providing high-quality delivery services. Together, these improvements in methodology have led to more generalizable results. Additionally, the use of observed availability of equipment and items instead of self-reported data during facility data collection increases the robustness of the readiness indicators and thus the accuracy of the relationship between the provision of delivery services and their use.

This study also has some limitations. One limitation is the temporal gap between the outcome variables and the service variables. Facility data reflect the "current" service environment at the time of the Haiti 2013 SPA, while use of facility delivery was measured over a 5-year time period preceding the 2012 Haiti DHS. Associating service provision and facility delivery use data could be problematic if the service environment changed substantially over this time period. Given the nature of delivery services, however, we do not expect that there was a substantial change in the availability of services and facilities' readiness to provide the service over the period.

While linking women to all of the facilities that they likely used reduces misclassification errors from the DHS GPS displacement procedure, the straight-line buffer approach does not take into account the mountainous terrain or the impassibility of roads during the rainy season, which may limit access to a linked facility. However, Nesbitt and colleagues compared 6 different measures of spatial access and found that the straightline linkage yields results similar to other geospatial algorithms in a developing-country setting. ${ }^{20}$ Finally, the buffer linkage between DHS clusters and SPA facilities may not be appropriate in areas where there is a high density of both health facilities and population, such as Haiti's metropolitan area. More precise measurements of the service environment are needed for such areas, as well as a better understanding of other drivers of use in areas where service availability may be less of an issue.

\section{CONCLUSION}

This study indicates the importance of improving physical access to delivery services in rural Haiti. Overall, health facilities in Haiti are poorly equipped and do not appear ready to provide high-quality delivery services. Improving the quality of care at health facilities could contribute to increased use of facility delivery particularly in nonmetropolitan urban areas, where $40 \%$ of women still deliver at home. Over the years, the global community has recognized the importance of providing women with quality maternal health services to reduce maternal mortality. ${ }^{38}$ After all, reducing maternal mortality by having women deliver in health facilities will only work if these facilities are ready to provide comprehensive obstetric care.

Acknowledgments: The authors acknowledge the financial support from the United States Agency for International Development (USAID) through the DHS Program.

Competing Interests: None declared.

\section{REFERENCES}

1. World Health Organization (WHO). Trends in Maternal Mortality: 1990 to 2015. Estimates by WHO, UNICEF, UNFPA, the World Bank and the United Nations Population Division. Geneva: WHO; 2015. http://www.who.int/reproductivehealth/publications/ monitoring/maternal-mortality-2015/en/. Accessed May 4, 2017. 
2. Anderson FWJ, Morton SU, Naik S, Gebrian B. Maternal mortality and the consequences on infant and child survival in rural Haiti. Matern Child Health J. 2007;1 1(4):395-401. CrossRef. Medline

3. Kirigia JM, Oluwole D, Mwabu GM, Gatwiri D, Kainyu LH. Effects of maternal mortality on gross domestic product (GDP) in the WHO African region. Afr J Health Sci. 2006;13(1-2):86-95. Medline

4. Cayemittes M, Busangu MF, Bizimana JdD, et al. Enquête Mortalité, Morbidité et Utilisation des Services, Haiiti, 2012. Calverton, Maryland: MSPP, IHE, and ICF International; 2013. https:// dhsprogram.com/pubs/pdf/FR273/FR273.pdf. Accessed May 4, 2017.

5. De Brouwere V, De Brouwere V, Tonglet R, Van Lerberghe W. Strategies for reducing maternal mortality in developing countries: what can we learn from the history of the industrialized West? Trop Med Int Health. 1998;3(10):771-782. CrossRef. Medline

6. Graham WJ, Bell JS, Bullough CHW. Can skilled attendance at delivery reduce maternal mortality in developing countries? In: Brouwere VD, Lerberghe V, Antwerp BIP, editors. Studies in Health Service Organization and Policy. Antwerp, Belgium: TIG Press; 2001.

7. Exavery A, Kanté A, Niozi M, et al. Access to institutional delivery care and reasons for home delivery in three districts of Tanzania. Int J Equity Health. 2014;13(1):48. CrossRef. Medline

8. Gabrysch S, Campbell OMR. Still too far to walk: literature review of the determinants of delivery service use. BMC Pregnancy Childbirth. 2009;9(1):34. CrossRef. Medline

9. Kitui J, Lewis S, Davey G. Factors influencing place of delivery for women in Kenya: an analysis of the Kenya Demographic and Health Survey, 2008/2009. BMC Pregnancy Childbirth. 2013;13(1):40. CrossRef. Medline

10. Montagu D, Yamey G, Visconti A, Harding A, Yoong J. Where do poor women in developing countries give birth? A multi-country analysis of demographic and health survey data. PLoS One. $2011 ; 6(2):$ e17155. CrossRef. Medline

11. Moyer CA, Dako-Gyeke P, Adanu RM. Facility-based delivery and maternal and early neonatal mortality in sub-Saharan Africa: a regional review of the literature. Afr J Reprod Health. 2013;17(3):30-43. Medline

12. Diamond-Smith N, Sudhinaraset M. Drivers of facility deliveries in Africa and Asia: regional analyses using the Demographic and Health Surveys. Reprod Health. 2015;12:6. CrossRef. Medline

13. Moyer CA, Mustafa A. Drivers and deterrents of facility delivery in sub-Saharan Africa: a systematic review. Reprod Health. 2013;10(1):40. CrossRef. Medline

14. Tey NP, Lai SL. Correlates of and barriers to the utilization of health services for delivery in South Asia and Sub-Saharan Africa. ScientificWorldJournal. 2013;2013:423403. CrossRef Medline

15. Thind A, Mohani A, Banerjee K, Hagigi F. Where to deliver? Analysis of choice of delivery location from a national survey in India. BMC Public Health. 2008;8(1):29. CrossRef. Medline

16. Babalola SO. Factors associated with use of maternal health services in Haiti: a multilevel analysis. Rev Panam Salud Publica. 2014;36(1):1-9. Medline

17. Séraphin MN, Ngnie-Teta I, Ayoya MA, et al. Determinants of institutional delivery among women of childbearing age in rural Haiti. Matern Child Health J. 2015;19(6):1400-1407. CrossRef. Medline

18. Kyei NNA, Campbell OMR, Gabrysch S. The influence of distance and level of service provision on antenatal care use in rural Zambia. PLoS One. 2012;7(10):e46475. CrossRef. Medline
19. Lohela TJ, Campbell OMR, Gabrysch S. Distance to care, facility delivery and early neonatal mortality in Malawi and Zambia. PLoS One. 2012;7(12):e521 10. CrossRef. Medline

20. Nesbitt RC, Gabrysch S, Laub A, et al. Methods to measure potentia spatial access to delivery care in low- and middle-income countries: a case study in rural Ghana. Int J Health Geogr. 2014;13(1):25. CrossRef. Medline

21. Skiles MP, Burgert CR, Curtis SL, Spencer J. Geographically linking population and facility surveys: methodological considerations. Popul Health Metr. 2013;1 1(1):14. CrossRef. Medline

22. Alexandre PK, Saint-Jean G, Crandall L, Fevrin E. Prenatal care utilization in rural areas and urban areas of Haiti. Rev Panam Salud Publica. 2005;18(2):84-92. Medline

23. Gage AJ, Guirlène Calixte M. Effects of the physical accessibility of maternal health services on their use in rural Haiti. Popul Stud (Camb). 2006;60(3):271-288. CrossRef. Medline

24. Ruktanonchai CW, Ruktanonchai NW, Nove A, et al. Equality in maternal and newborn health: modelling geographic disparities in utilisation of care in five East African countries. PloS One. 2016;11(8):e0162006. CrossRef. Medline

25. Choulagai B, Onta S, Subedi N, et al. Barriers to using skilled birth attendants services in mid- and far-western Nepal: a cross-sectional study. BMC Int Health Hum Rights. 2013;13(1):49. CrossRef. Medline

26. Kinney MV, Kerber KJ, Black RE, et al; Science in Action: Saving the lives of Africa's Mothers, Newborns, and Children working group. Sub-Saharan Africa's mothers, newborns, and children: where and why do they die? PLoS Med. 2010;7(6):e1000294. CrossRef. Medline

27. Yaffee AQ, Whiteside LK, Oteng RA, et al. Bypassing proximal health care facilities for acute care: a survey of patients in a Ghanaian Accident and Emergency Centre. Trop Med Int Health. 2012;17(6):775-781. CrossRef. Medline

28. Karkee R, Lee AH, Pokharel PK. Women's perception of quality of maternity services: a longitudinal survey in Nepal. BMC Pregnancy Childbirth. 2014;14(1):45. CrossRef. Medline

29. Acharya LB, Cleland J. Maternal and child health services in rural Nepal: does access or quality matter more? Health Policy Plan. 2000;15(2):223-229. CrossRef. Medline

30. Burgert CR, Colston J, Roy T, Zachary B. Geographic displacement procedure and georeferenced data release policy for the Demographic and Health Surveys. DHS Statistical Analysis Reports No. 7. Calverton, Maryland: ICF International; 2013. https:// dhsprogram.com/pubs/pdf/SAR7/SAR7.pdf. Accessed May 4, 2017.

31. Ministère de la Santé Publique et de la Population. Plan Directeur de la Santé 2012-2022. Port-au-Prince, Haïti: MSPP; 2013.

32. World Health Organization (WHO). Service Availability and Readiness Assessment (SARA): An Annual Monitoring System for Service Delivery. Geneva: WHO; 2013. http://www.who.int/ healthinfo/systems/SARA_Reference_Manual_Full.pdf. Accessed May 4, 2017.

33. Raudenbush SW, Bryk AS. Hierarchical Linear Models: Applications and Data Analysis Methods. London: Sage Publications; 2002.

34. Moyer CA, Mustafa A. Drivers and deterrents of facility delivery in sub-Saharan Africa: a systematic review. Reprod Health. 2013;10(1):40. CrossRef. Medline

35. Gabrysch S, Cousens S, Cox J, Campbell OMR. The influence of distance and level of care on delivery place in rural Zambia: a study of linked national data in a geographic information system. PLoS Med. $2011 ; 8(1):$ e1000394. CrossRef. Medline

36. Stekelenburg J, Kyanamina S, Mukelabai M, Wolffers I, Roosmalen J. Waiting too long: low use of maternal health services in Kalabo, 
Zambia. Trop Med Int Health. 2004;9(3):390-398. CrossRef. Medline

37. Harvey S, Blandón YC, McCaw-Binns A, et al; Nicaraguan Maternal and Neonatal Health Quality Improvement Group. Are skilled birth attendants really skilled? A measurement method, some disturbing results and a potential way forward. Bull World Health Organ. 2007;85(10):783-790. CrossRef. Medline

38. Koblinsky M, Moyer CA, Calvert C, et al. Quality maternity care for every woman, everywhere: a call to action. Lancet. 2016;388 (10057):2307-2320. CrossRef Medline

\section{Peer Reviewed}

Received: 2016 Oct 10; Accepted: 2017 Mar 13; First Published Online: 2017 May 25

Cite this article as: Wang W, Winner M, Burgert-Brucker CR. Limited service availability, readiness, and use of facility-based delivery care in Haiti: a study linking health facility data and population data. Glob Health Sci Pract. 2017;5(2):244-260. https://doi.org/10.9745/GHSP-D-16-0031 1

(c) Wang et al. This is an open-access article distributed under the terms of the Creative Commons Attribution License, which permits unrestricted use distribution, and reproduction in any medium, provided the original author and source are properly cited. To view a copy of the license, visit http:// creativecommons.org/licenses/by/3.0/. When linking to this article, please use the following permanent link: https://doi.org/10.9745/GHSP-D-1600311 
